\title{
Targeted knockdown of death-associated protein kinase expression induces TRAIL-mediated apoptosis in human endometrial adenocarcinoma cells
}

\author{
TAO BAI $^{1}$, TETSUJI TANAKA ${ }^{1}$ and KAZUNORI YUKAWA ${ }^{2}$ \\ ${ }^{1}$ Department of Obstetrics and Gynecology, Wakayama Medical University, 811-1 Kimi-idera, \\ Wakayama 641-0012; ${ }^{2}$ Department of Physiology, Faculty of Pharmacy, Meijo University, \\ 150 Yagotoyama, Tempaku-ku, Nagoya 468-8503, Japan
}

Received January 26, 2010; Accepted March 30, 2010

DOI: 10.3892/ijo_00000668

\begin{abstract}
Death-associated protein kinase (DAPK) is a serine/threonine kinase that participates in the modulation of apoptosis and tumor suppression. Our previous study revealed high levels of DAPK protein expression in differentiated endometrial adenocarcinoma cells. To clarify the role of DAPK in human endometrial adenocarcinomas, we downregulated endogenous DAPK expression in HHUA cells, a well-differentiated endometrial adenocarcinoma cell line, using specific small-interfering RNAs (siRNAs). The suppression of endogenous DAPK expression triggered apoptosis in HHUA cells, as evidenced by an increase in the sub-G1 DNA content in flow cytometric analyses. The apoptosis induced by the DAPK siRNA transfections was caspase-dependent, as characterized by the activations of caspase- $3,-8$ and -9 . RNase protection assays detected higher levels of tumor necrosis factor-related apoptosis-inducing ligand (TRAIL), DR4 and DR5 transcripts in the DAPK siRNA-transfected HHUA cells than in the control siRNA-transfected cells. Consistent with these findings, enzyme-linked immunosorbent assays revealed that the DAPK siRNA transfections significantly increased the secretion of TRAIL protein from the cells. Treatment with recombinant human TRAIL protein dose-dependently suppressed the cell viability of HHUA cells. The present findings reveal that down-regulation of endogenous DAPK expression in HHUA cells induces caspasedependent apoptosis, possibly through increased TRAIL, DR4 and DR5 signaling, thereby suggesting that DAPK expression is essential for HHUA cell survival. Consequently, endogenous DAPK mRNA may represent a potential candidate for molecularly targeted anticancer therapies.
\end{abstract}

Correspondence to: Dr Tetsuji Tanaka, Department of Obstetrics and Gynecology, Wakayama Medical University, 811-1 Kimi-idera, Wakayama 641-0012, Japan

E-mail: obgywmu@wakayama-med.ac.jp

Key words: death-associated protein kinase, TRAIL, endometrial adenocarcinoma, siRNA

\section{Introduction}

Endometrial adenocarcinomas are the most common gynecological malignancies. The development of these cancers results from a series of genetic alterations that transform the normal endometrium through hyperplasia and dysplasia to culminate in overt carcinoma. The first-line treatment for patients with early endometrial cancers is radical surgical resection. For advanced endometrial cancers, radiotherapy and/or chemotherapy are usually combined with the surgical treatment. Chemotherapy is applied at first for unresectable advanced endometrial cancer patients and after surgery, radiotherapy or chemotherapy for recurrent endometrial cancer patients. However, when patients have been treated with repeated chemotherapy regimens, their cancer cells can become multidrug-resistant to typical anticancer drugs. Such multidrug-resistant endometrial cancer patients require novel molecularly targeted anticancer therapies to induce specific cell death in endometrial cancer tissues. The targeted molecules must be important survival factors for endometrial adenocarcinoma cells but not essential factors for individual human survival. However, there have been no reports of the identification of molecules that can cause specific endometrial adenocarcinoma cell death and that do not induce any severe adverse effects in humans.

Apoptosis is crucial for embryonic development and maintenance of tissue homeostasis. The signaling pathways leading to apoptosis are comprised of both extrinsic and intrinsic pathways. The extrinsic pathway involves the engagement of members of the tumor necrosis factor receptor (TNFR) family, also known as the death receptor family. These receptors bind to their specific ligands and recruit Fas-associated death domain (FADD) and procaspase- 8 to assemble the death-inducing signaling complex (DISC), thereby resulting in the activation of caspases, including caspase- 3 and -8 , which induce apoptosis (1). Tumor necrosis factor-related apoptosis-inducing ligand (TRAIL) is a member of the TNF family $(2,3)$. Similar to the well-documented interplay between Fas ligand and Fas that activates caspase- 8 to directly elicit apoptosis, TRAIL binds to its specific death receptors, DR4 and DR5, to induce apoptosis, while the expressions of its decoy receptors, DcR1 and DcR2, antagonize 
Table I. DAPK siRNA sequences.

\begin{tabular}{lc}
\hline DAPK siRNA & siRNA sequence \\
\hline DAPK siRNA 001 & 5'- CAACAUCAUGCAAAGUGAAACAGUU-AG-3' \\
& 3'-AU-GUUGUAGUACGUUUCACUUUGUCAA-5' \\
DAPK siRNA 002 & 5'-AGCCAAGAAUUAAGCUCAAGCUGUU-AG-3' \\
& 3'-AU-UCGGUUCUUAAUUCGAGUUCGACAA-5'
\end{tabular}

TRAIL-mediated apoptosis (4-7). In addition, TRAIL appears to be selectively cytotoxic toward tumor cells without exhibiting appreciable cytotoxicity toward normal cells (8-12). The intrinsic pathway of apoptosis is triggered in response to chemotherapeutic drugs, and involves the formation of apoptosomes composed of cytochrome c released from mitochondria as well as apoptotic protease-activating factor-1 (Apaf-1) and procaspase-9, in which caspase-9 is cleaved to further promote the activation of caspase- 3 that leads to apoptosis (13). Recent reports have also shown that active caspase- 8 has the ability to process the proapoptotic Bcl-2 family member Bcl-2-interacting domain (BID) to truncated BID (tBID) to further promote cytochrome c release, thereby linking the extrinsic pathway to the intrinsic pathway $(14,15)$.

Death-associated protein kinase (DAPK) is a $\mathrm{Ca}^{2+} /$ calmodulin-dependent serine/threonine kinase that functions as a positive mediator of apoptosis triggered by interferon (IFN)- $\gamma$, TNF- $\alpha$, anti-Fas antibodies, transforming growth factor- $\beta$, c-myc and E2F oncogenes, ceramide and detachment from the extracellular matrix (16-23). Moreover, loss of DAPK expression has been implicated in tumorigenesis and metastasis $(24,25)$, thus suggesting a crucial role for DAPK in the apoptotic process under pathological conditions. On the other hand, several lines of evidence have indicated that DAPK may have an antiapoptotic function. Inhibition of DAPK expression in HeLa cells, 3T3 fibroblasts and primary human vascular smooth muscle cells with an antisense DAPK was found to increase apoptosis $(26,27)$. These studies support the notion that DAPK plays cytoprotective roles under certain cell survival conditions.

In a previous study, we detected higher DAPK protein expression levels in differentiated endometrial adenocarcinoma cells than in normal primary endometrial cells and several ovarian and uterine carcinoma cells including HeLa cells (28). These results suggest that DAPK regulates cell survival or apoptosis of human endometrial adenocarcinoma cells. When we bred DAPK-mutant mice for several years to investigate their nervous systems and ovarian functions, we observed that the DAPK-mutant mice showed similar survival rates to normal adult mice and became pregnant and had offspring (unpublished data). Taken together, these results suggest that DAPK is neither a lethal molecule for an individual nor an essential molecule for reproduction. Therefore, we examined whether DAPK represents a candidate molecule for a targeted anticancer therapy for advanced cancer patients with differentiated human endometrial adenocarcinoma in which DAPK protein is highly expressed. To achieve this, we utilized specific DAPK small-interfering RNAs (siRNAs) to knock down endogenous DAPK expression in HHUA cells (29) to directly investigate the role of endogenous DAPK in endometrial adenocarcinoma cell survival.

\section{Materials and methods}

Cell line and culture. The HHUA cell line (29) was obtained from Riken Cell Bank (Tsukuba, Japan). The cells were cultured in OPTI-MEM (Invitrogen Corp., Carlsbad, CA) supplemented with 5\% fetal bovine serum (FBS) (Equitech Bio Inc., Ingram, TX), penicillin (100 U/ml), streptomycin (100 U/ml) and Fungizone $(0.25 \mu \mathrm{g} / \mathrm{ml}$; Invitrogen Corp.) in the presence of $5 \% \mathrm{CO}_{2}$ and $95 \%$ air at $37^{\circ} \mathrm{C}$.

Transfection of DAPK siRNAs. Two DAPK siRNA duplexes were designed and synthesized by iGENE Therapeutics Inc. (Tsukuba, Japan). The siRNA sequences are shown in Table I. A negative control siRNA was purchased from Ambion Inc. (Austin, TX). Lipofectamine 2000 (Invitrogen Corp.) was used as the transfection reagent according to the manufacturer's instructions. For experiments, cells were seeded in 6-well plates $\left(2.5 \times 10^{5}\right.$ cells/well $)$ or $10-\mathrm{cm}$ dishes $\left(2 \times 10^{6}\right.$ cells/dish $)$, cultured for $24 \mathrm{~h}$ and then transfected with the DAPK siRNAs or control siRNA at a final concentration of $50 \mathrm{nM}$. Subsequently, the cells were cultured for $24-48 \mathrm{~h}$ for mRNA analyses and 48-72 $\mathrm{h}$ for protein assays before being harvested as indicated.

RNA isolation and real-time RT-PCR. Total RNA was isolated from the cultured cells using an RNeasy Mini kit (Qiagen Inc., Valencia, CA) for real-time RT-PCR and RNase protection assays. For real-time RT-PCR, $1 \mu \mathrm{g}$ of total RNA was predigested with DNase I (Invitrogen Corp.) and subjected to cDNA synthesis using a reverse transcriptase kit (Bio-Rad, Hercules, CA) in a $20-\mu 1$ reaction mixture. The cDNA product was diluted to $100 \mu 1$. Real-time PCR amplifications were performed using an iCycler (Bio-Rad). Each PCR mixture $(25 \mu \mathrm{l})$ contained $2.5 \mu \mathrm{l}$ of diluted cDNA, $12.5 \mu \mathrm{l}$ of $\mathrm{iQ}^{\mathrm{TM}}$ SYBR Green Supermix (Bio-Rad) and $0.5 \mu \mathrm{M}$ primers. The PCR amplifications were performed with specific primers for DAPK (forward, 5'-GATAGAAATGTCCCCAAACC TCG-3'; reverse, 5'-TCTTCTTGGATCCTTGACCAGAA-3') to amplify a 343-bp product. Actin was amplified as a positive control. For each analysis, an initial hot start at $95^{\circ} \mathrm{C}$ for $3 \mathrm{~min}$ was followed by 40 cycles of amplification $\left(95^{\circ} \mathrm{C}\right.$ for $30 \mathrm{sec}, 55^{\circ} \mathrm{C}$ for $30 \mathrm{sec}$ and $72^{\circ} \mathrm{C}$ for $\left.60 \mathrm{sec}\right)$. The DAPK mRNA levels in the DAPK siRNA-treated cells relative to the control siRNA-transfected cells were calculated based on the 
$2^{-\Delta \Delta C T}$ method, in which $\Delta \Delta \mathrm{C}_{\mathrm{T}}=\Delta \mathrm{C}_{\mathrm{T} \text { DAPK siRNA }}-\Delta \mathrm{C}_{\mathrm{T} \text { control siRNA }}=$ $\left(\mathrm{C}_{\mathrm{T} \text { DAPK }}-\mathrm{C}_{\mathrm{T} \text { actin }}\right)_{\mathrm{DAPK} \text { siRNA }}-\left(\mathrm{C}_{\mathrm{T} \text { DAPK }}-\mathrm{C}_{\mathrm{T} \text { actin }}\right)_{\text {control siRNA }}$.

Western blotting. For Western blotting analysis, the cells were collected at 48-72 $\mathrm{h}$ after transfection with the DAPK siRNAs or control siRNA, and lysed in phosphate-buffered saline containing $1 \% \mathrm{NP}-40,0.1 \%$ sodium dodecyl sulfate, Complete protease inhibitor cocktail (Roche Diagnostics Corp., Indianapolis, IN) and $1 \mathrm{mM}$ phenylmethyl sulfonyl fluoride. The protein concentrations of the cell lysates were quantified by Coomassie Plus Protein assays (Pierce Biotechnology Inc., Rockford, IL). Equal amounts of the total proteins were separated by SDS-PAGE using a $7.5 \%$ gel, and then transferred to a polyvinylidene fluoride membrane (ATTO Corp., Tokyo, Japan). After sequential incubations with primary and secondary antibodies, the immunocomplexes on the membranes were detected using enhanced chemiluminescence (ECL) or ECL plus kits (Amersham Pharmacia Biotech, Uppsala, Sweden). The antibodies used were purchased from the following sources: mouse monoclonal anti-DAPK antibody (Sigma, St. Louis, MO); mouse monoclonal anti-cleavedcaspase-3 antibodies (Cell Signaling Technology, Beverly, MA); mouse monoclonal anti-caspase- 8 antibody (Merck Bioscience Inc., San Diego, CA); and mouse monoclonal anti-caspase-9 antibody (R\&D Systems Inc., Minneapolis, $\mathrm{MN})$. The membranes were stripped and reprobed with an anti- $\beta$-actin antibody (Sigma).

Flow cytometric DNA analysis. Quantification of apoptotic cell death was carried out by flow cytometry (FACS) analysis of the sub-G1 DNA content. Cells were treated as described above. After 48-72 h of culture, the cells were collected, washed and fixed in $70 \%$ ethanol at $-20^{\circ} \mathrm{C}$. The cells were then stained with PI/RNase staining buffer (BD PharMingen, San Diego, CA) for $15 \mathrm{~min}$ at room temperature. Nuclei from apoptotic cells were quantified by flow cytometry using a FACSCalibur and the associated CellQuest program (BD Biosciences, Mountain View, CA). Values were calculated as the means $\pm \mathrm{SD}$ of three independent experiments.

Caspase activity assay. The caspase-3/7, -8 and -9 activities in the DAPK siRNA-treated or control siRNA-transfected HHUA cells were measured using Caspase-Glo ${ }^{\mathrm{TM}}-3 / 7,-8$ and -9 Assay kits (Promega Corp., Madison, WI) according to the manufacturer's instructions with some previously described modifications (1). Both adherent and floating cells were collected and lysed in phosphate-buffered saline containing $1 \%$ NP-40, $0.1 \%$ sodium dodecyl sulfate, complete protease inhibitor cocktail and $1 \mathrm{mM}$ phenylmethyl sulfonyl fluoride. After centrifugation, 10 or $20 \mu \mathrm{g}$ of protein in a total volume of $50 \mu \mathrm{l}$ was mixed with $50 \mu \mathrm{l}$ of equilibrated CaspaseGlo $^{\mathrm{TM}}-3 / 7,-8$ or -9 reagent and incubated for $1 \mathrm{~h}$ at room temperature. Subsequently, the luminescence was measured using a BLR-301 Luminescence Reader (Aloka Corp., Tokyo, Japan). The caspase activities were calculated based on standard curves generated using human recombinant caspase-3, -8 and -9 (Biomol Research Laboratories Inc., Plymouth Meeting, PA), respectively.

Cell viability assay. The responses of HHUA cells to soluble human recombinant TRAIL (Biomol Research Laboratories
Inc.) were measured as follows. HHUA cells were plated in 96-well plates at $5 \times 10^{3}$ cells/well $(n=6)$ and incubated with various concentrations of TRAIL for 48-72 h. At the end of the treatments, the viable cell numbers were determined using a Cell Counting kit (Dojindo Chemical Laboratory Co. Ltd., Tokyo, Japan) according to the manufacturer's instructions. The absorbances at $450 \mathrm{~nm}$ were measured using a microplate reader. A viability of $100 \%$ was defined as the absorbance obtained for cells without TRAIL treatment.

Ribonuclease protection assay. A multiprobe RNase protection assay was performed using a RiboQuant ${ }^{\mathrm{TM}}$ hAPO-3d multiprobe template set and an RNase protection assay (RPA) kit (BD PharMingen) according to the manufacturer's protocol. Biotin-conjugated probes were prepared using a Non-Rad In Vitro Transcription kit (BD PharMingen). The probes were combined with $10 \mu \mathrm{g}$ of total RNA isolated from DAPK siRNA-transfected or control siRNA-transfected HHUA cells. After denaturation at $95^{\circ} \mathrm{C}$ for $3 \mathrm{~min}$, the mixtures were hybridized at $56^{\circ} \mathrm{C}$ for $12-16 \mathrm{~h}$ before RNase digestion. RNA hybrids were separated in $4.75 \%$ acrylamide/ $8 \mathrm{M}$ urea denaturing gels, and the protected fragments were transferred to positively charged nylon membranes. The fragments on the membranes were detected using the RPA detection kit and the protected fragments were quantified with a luminocapture apparatus and lane analyzer software (ATTO Corp.). The relative expression of each mRNA species was calculated after normalization by the expression level of the housekeeping gene L32.

Enzyme-linked immunosorbent assay (ELISA) of TRAIL. DAPK siRNA-transfected or control siRNA-transfected HHUA cells were collected at various time-points and lysed in lysis buffer containing a protease inhibitor provided in a Function ELISA $^{\mathrm{TM}}$ TRAIL/Apo2L kit (Active Motif, Carlsbad, CA). The TRAIL assay was performed according to the manufacturer's instructions. Briefly, $100 \mu \mathrm{g}$ of cell lysates or standard recombinant TRAIL at known concentrations were added to wells coated with a capture antibody specific for TRAIL and incubated for $4 \mathrm{~h}$. After washing, a detection antibody was added to the wells and incubated for $60 \mathrm{~min}$. After further washing, an alkaline phosphatase (ALP)conjugated secondary antibody was added to the wells and incubated for $60 \mathrm{~min}$. After five washes, a substrate solution for ALP was added to induce the formation of a colored reaction product. The intensities of the colored product in the wells were determined by measuring the absorbances at $405 \mathrm{~nm}$ using a spectrophotometer.

Statistical analysis. The data are expressed as means \pm SD. Comparisons between experimental groups were performed by analysis of variance (ANOVA). If the ANOVA was significant, post-hoc comparisons were conducted using Scheffe's test. The level of statistical significance was set at $p<0.05$.

\section{Results}

Specific down-regulation of endogenous DAPK expression by DAPK siRNA transfections induces apoptosis in HHUA cells. We previously reported that endogenous DAPK protein was highly expressed in the well-differentiated endometrial 


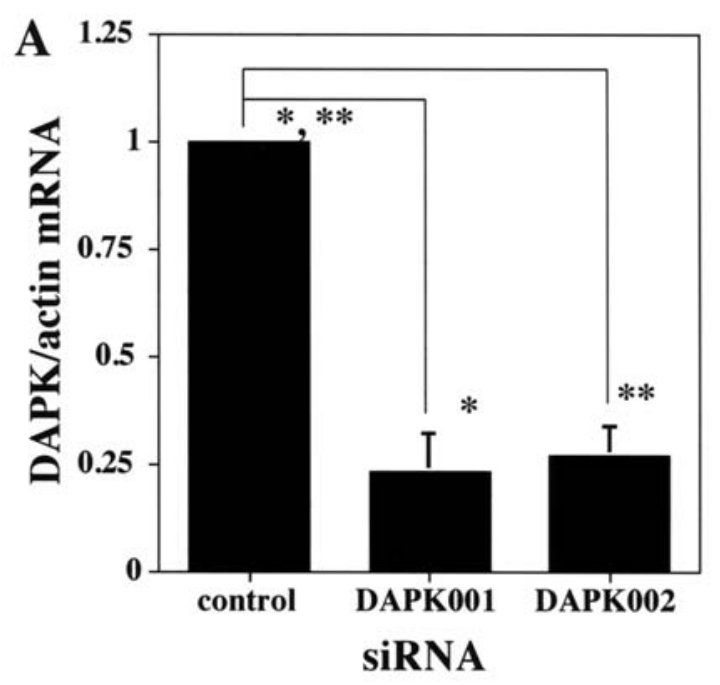

B

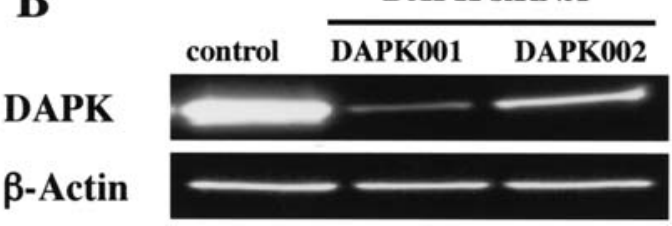

Figure 1. Down-regulation of endogenous DAPK expression in the DAPK siRNA-transfected HHUA cells. (A) Real-time RT-PCR analyses confirm that the DAPK mRNA levels are significantly reduced in the DAPK siRNAtransfected HHUA cells (DAPK001 and DAPK002) compared with the control siRNA-transfected cells following normalization by the B-actin mRNA levels; ${ }^{*}{ }^{* *} \mathrm{p}<0.05$. (B) Western blot analyses show decreased DAPK protein expression levels in the DAPK siRNA-transfected HHUA cells (DAPK001 and DAPK002) compared with the control siRNA-transfected cells.

adenocarcinoma cell line HHUA compared with normal endometrial tissues. To investigate the role of endogenous DAPK protein in HHUA cells, we performed siRNA transfection experiments using two double-stranded siRNAs (DAPK
siRNAs 001 and 002) against human DAPK mRNA to examine the effects of specific down-regulation of DAPK expression in HHUA cells. Real-time RT-PCR analyses confirmed that transient transfections of the DAPK siRNA sequences into HHUA cells significantly decreased the DAPK mRNA expression levels by $\sim 75 \%$ after $24 \mathrm{~h}$ relative to the levels in the control siRNA-transfected cells (Fig. 1A). Similarly, Western blot analyses revealed that DAPK protein expression was strongly suppressed in DAPK siRNAtransfected HHUA cells at $48 \mathrm{~h}$ after transfection (Fig. 1B). No non-specific inhibitory effects were detected for $\beta$-actin protein expression as an internal control.

Next, we examined the DAPK siRNA-transfected and control siRNA-transfected HHUA cells for the effects of the reduced endogenous DAPK expression. Many of the DAPK siRNA-transfected cells became detached from the substratum, whereas the control siRNA-transfected cells did not (Fig. 2), suggesting that reduced DAPK expression led to reduced cell adhesion and/or cell death induction in HHUA cells. To clarify these possibilities, the sub-G1 DNA contents in the DAPK siRNA-transfected and control siRNA-transfected cells were evaluated by flow cytometric analyses. Significantly higher percentages of sub-G1 DNA contents were detected in the DAPK siRNA-transfected cells compared with the control siRNA-transfected cells (54.1 and $26.23 \%$ vs. 5.98\%) (Fig. 3). These data indicate that DAPK siRNAmediated knockdown of endogenous DAPK expression induces cell death in HHUA cells.

DAPK siRNA-induced cell death in HHUA cells is caspasedependent. It is well documented that apoptosis is mediated by a cascade of caspases, which are further divided into initiators or effectors of apoptosis such as caspase-3, -8 and -9 . Therefore, we examined whether procaspase- $3,-8$ and -9 were activated in HHUA cells after transfection of the DAPK siRNAs. First, we conducted Western blot analyses to examine caspase activation. As shown in Fig. 4, cleaved fragments of
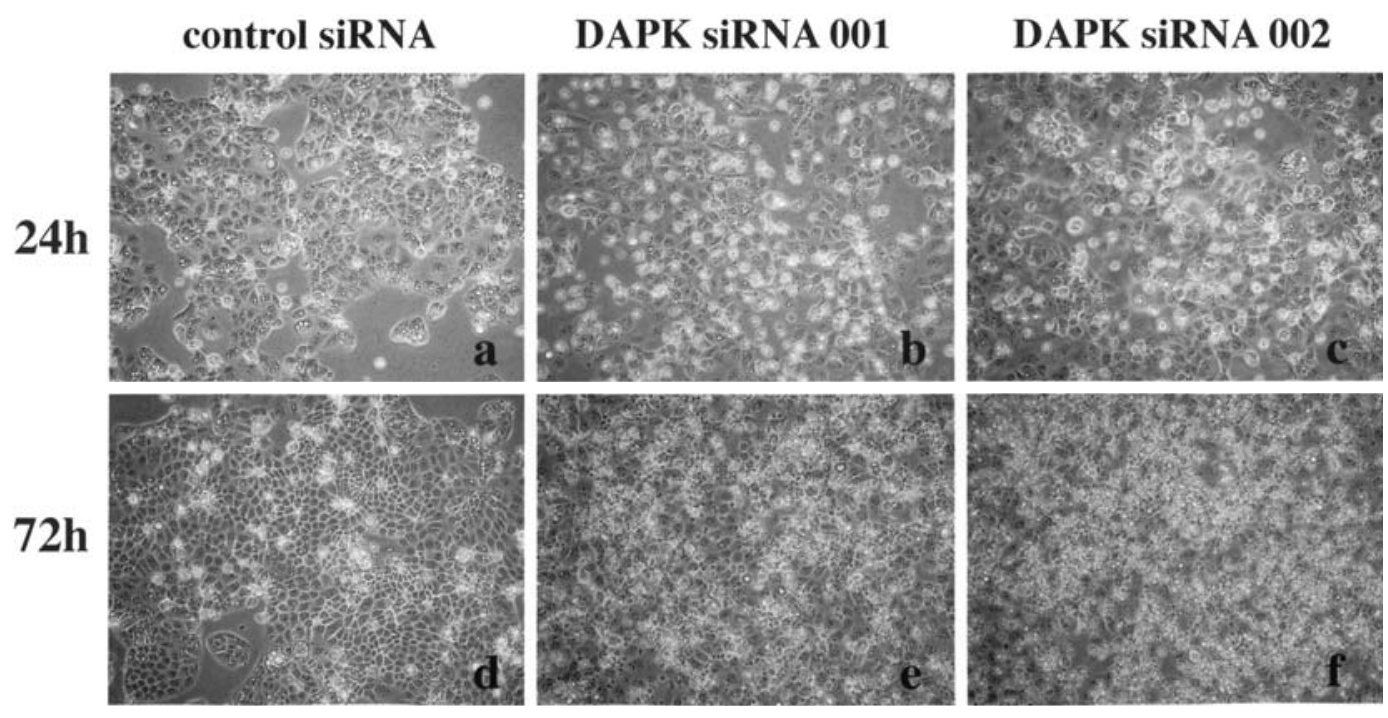

Figure 2. Phase-contrast microscopic findings for the DAPK siRNA-transfected and control siRNA-transfected HHUA cells. The images show the microscopic findings for HHUA cells at $24 \mathrm{~h}$ (a-c) and $72 \mathrm{~h}$ (d-f) after the siRNA transfections. HHUA cells transfected with the control siRNA (a and d), DAPK siRNA 001 (b and e) and DAPK siRNA 002 (c and f) are shown. In the DAPK siRNA-transfected cells, increased cell detachment from the culture dishes is observed. 


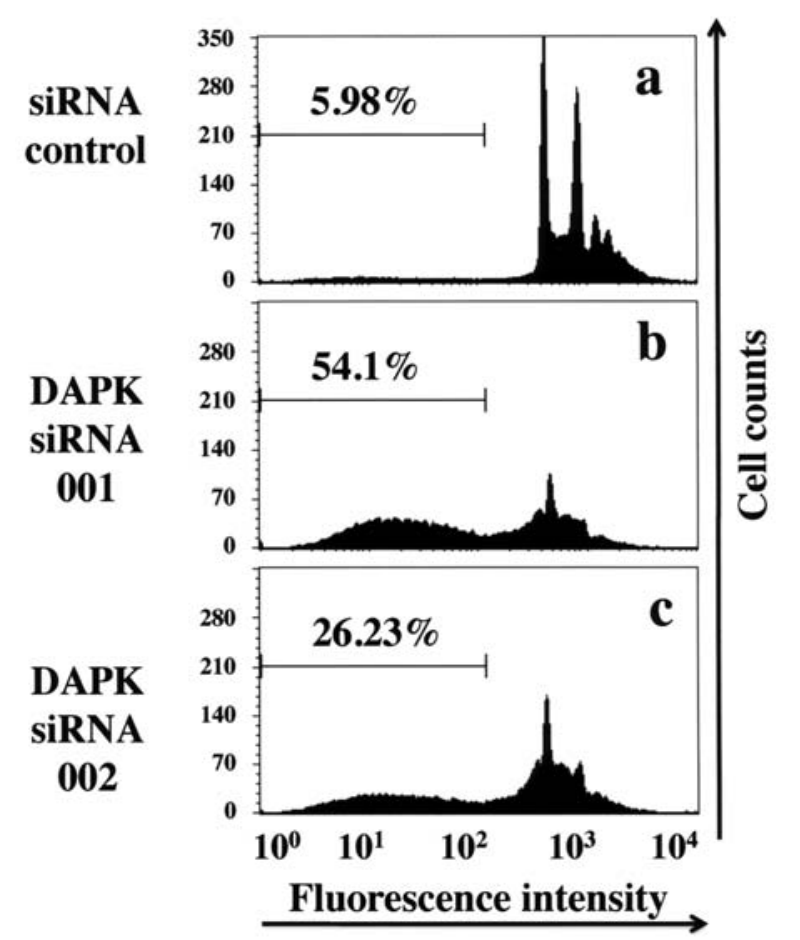

Figure 3. Flow cytometric DNA analyses of the DAPK siRNA-transfected HHUA cells. The panels show representative data from one of three flow cytometric assays. The sub-G1 contents indicating DNA fragmentation are apparently higher in the HHUA cells transfected with DAPK siRNA 001 (b) and DAPK siRNA 002 (c) than in the cells transfected with the control siRNA (a).

caspase-3, -8 and -9 were strongly enhanced in the DAPK siRNA-treated HHUA cells compared with the control siRNAtreated cells. Next, the DAPK siRNA-treated and control siRNA-treated cells were subjected to comparative caspase activity assays. Consistent with the results of the Western

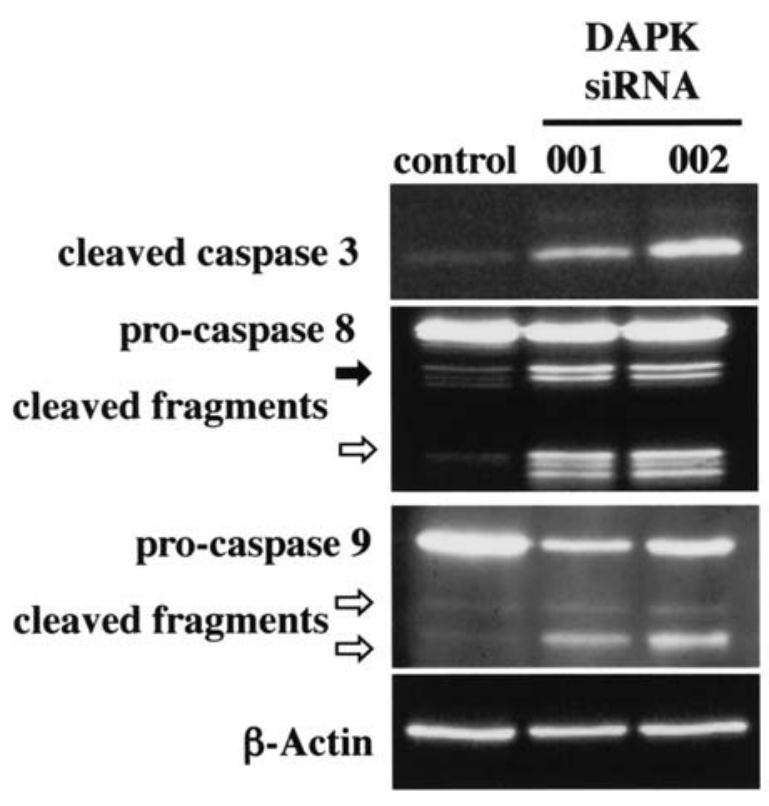

Figure 4. Western blot analyses of caspase- $3,-8$ and -9 in the DAPK siRNAtransfected HHUA cells. The suppression of endogenous DAPK protein expression in the DAPK siRNA-transfected cells triggers the cleavage of caspase- $3,-8$ and -9 .

blot analyses, the DAPK siRNA-treated cells showed much higher levels of caspase-3, -8 and -9 activities than the control siRNA-transfected cells (Fig. 5). Taken together, these data indicate that knockdown of endogenous DAPK by DAPKspecific siRNAs triggers caspase-dependent apoptosis in HHUA cells. Alternatively, DAPK may be an essential molecule for maintaining the cell survival of HHUA cells.

Endogenous DAPK expression in HHUA cells regulates the mRNA expressions of apoptosis-related genes. To elucidate
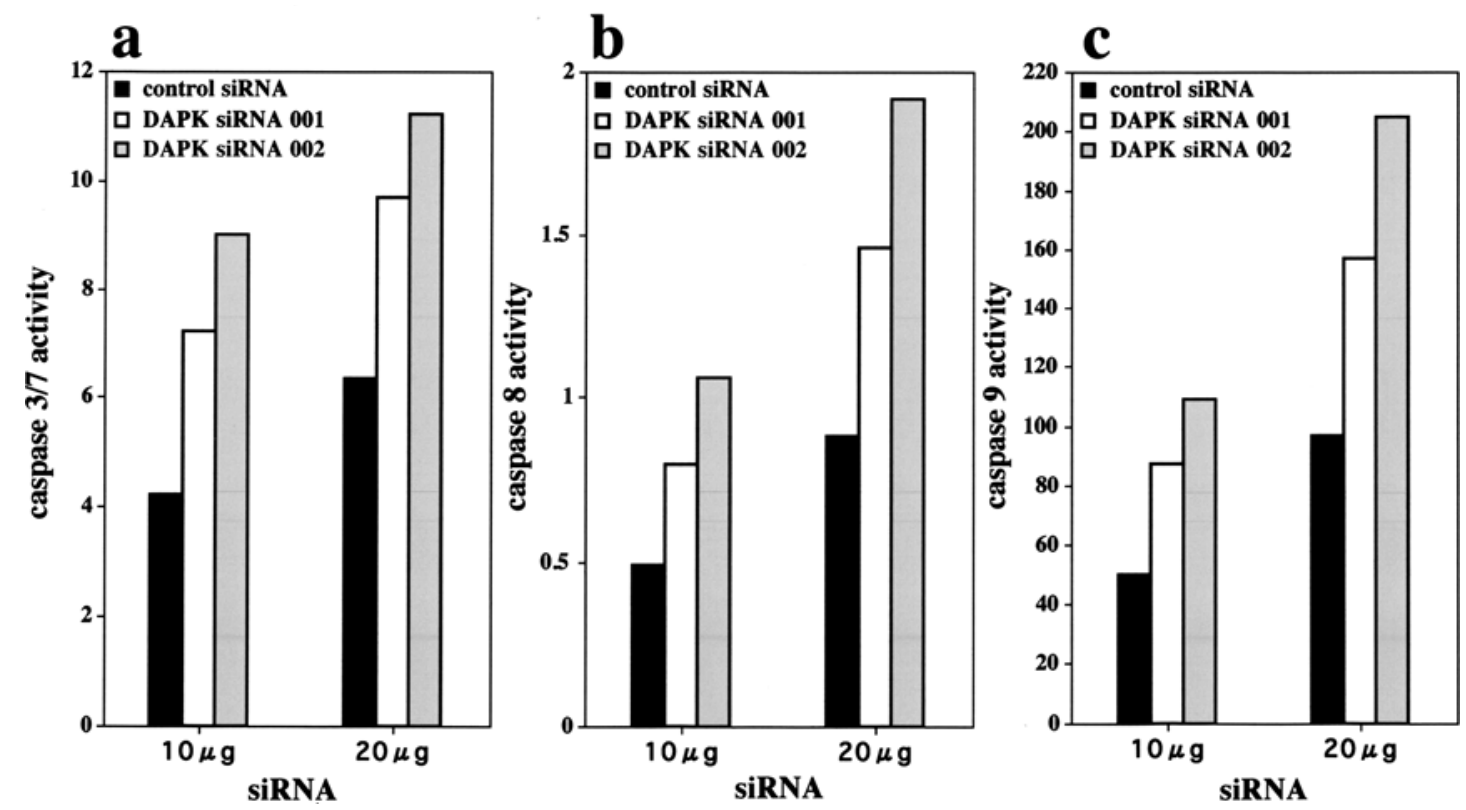

Figure 5. Caspase activity assays for caspase-3, -8 and -9 in the DAPK siRNA-transfected HHUA cells. The caspase activity assays reveal enhanced activities of caspase-3 (a), -8 (b) and -9 (c) in the DAPK siRNA-transfected cells compared with the control siRNA-transfected cells. Closed bars, siRNA-transfected cells; open bars, DAPK siRNA 001-transfected cells; gray bars, DAPK siRNA 002-transfected cells. 


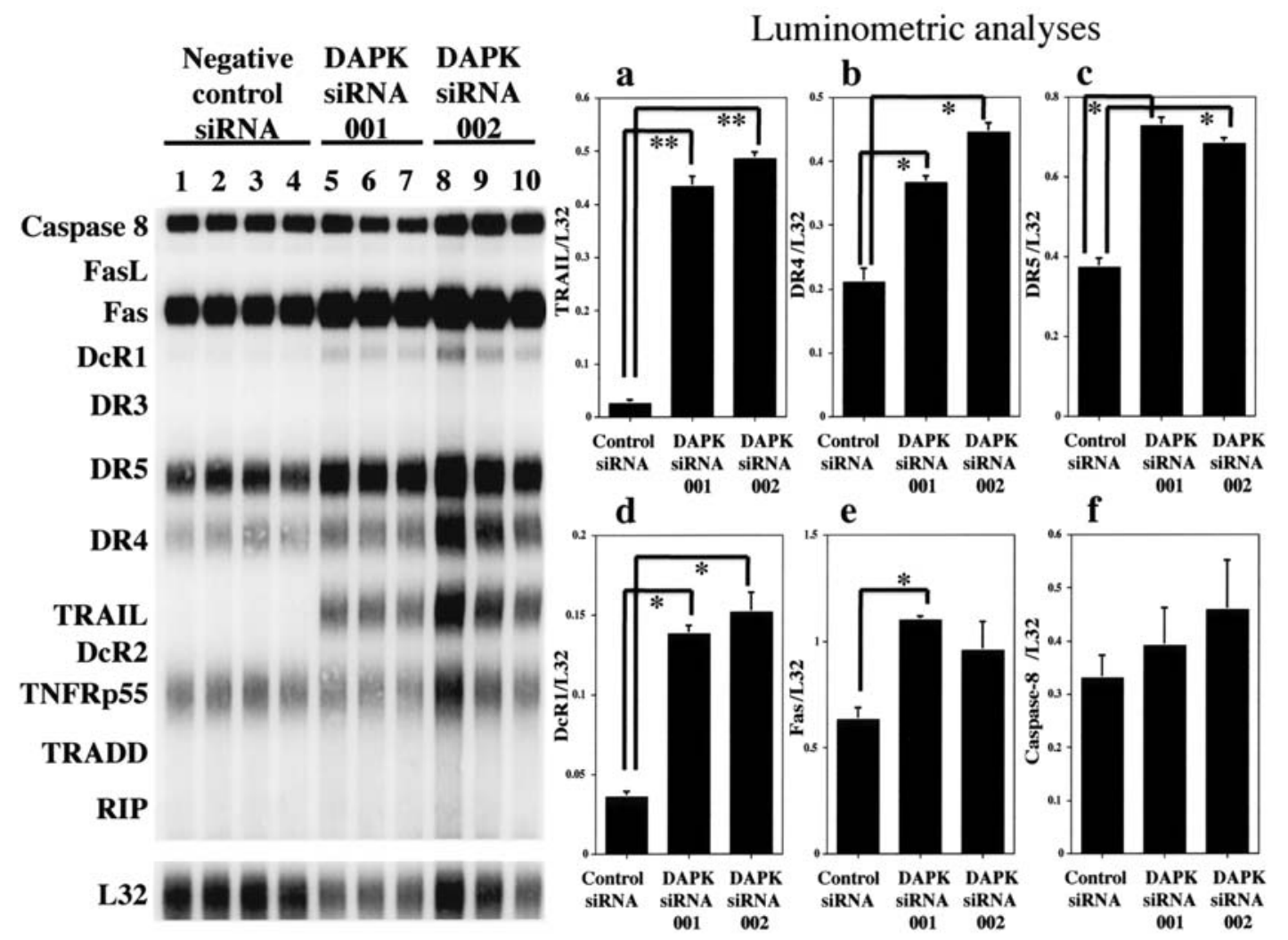

Figure 6. RNase protection assays for the effects of the DAPK siRNA transfections on apoptosis-related gene expressions in HHUA cells. The DAPK siRNAtransfected cells show increased mRNA expressions for Fas, DcR1, DR5, DR4 and TRAIL compared with the control siRNA-transfected cells. The left panel shows electrophoretic data for the siRNA-transfected cells. Lanes 1-4, control siRNA-transfected cells $(\mathrm{n}=4)$; lanes 5-7, DAPK siRNA 001-transfected cells $(\mathrm{n}=3)$; lanes 8-10, DAPK siRNA 002-transfected cells $(\mathrm{n}=3)$. The right panels shows luminometric analyses of the relative mRNA expression levels against control L32 mRNA. siRNA1, DAPK siRNA 001; siRNA2, DAPK siRNA 002. (a) TRAIL/L32 ratio, (b) DR4/L32 ratio, (c) DR5/L32 ratio, (d) DcR1/L32 ratio, (e) Fas/L32 ratio, (f) caspase-8/L32 ratio; ${ }^{*} \mathrm{p}<0.05,{ }^{* *} \mathrm{p}<0.01$.
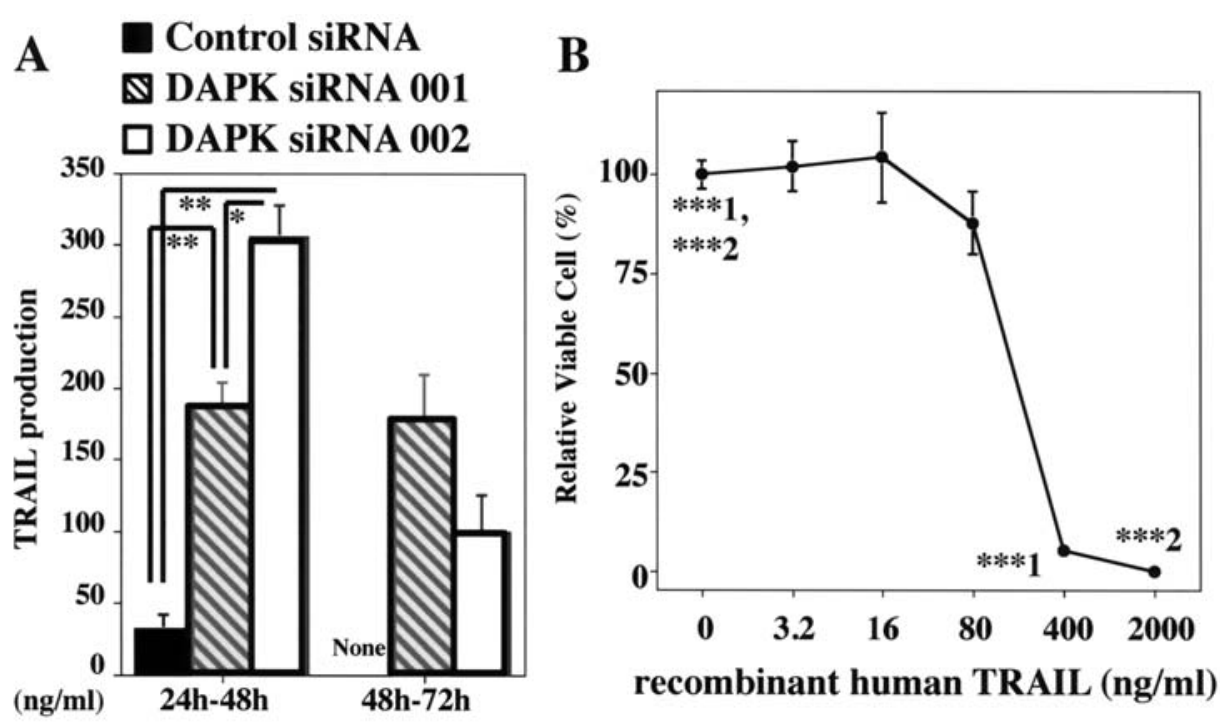

Figure 7. Secretion and effects of TRAIL in the DAPK siRNA-transfected HHUA cells. (A) The DAPK siRNA-transfected cells show increased TRAIL protein secretion into the culture media. Black bars, HHUA cells transfected with $50 \mathrm{nM}$ control siRNA; hatched bars, HHUA cells transfected with $50 \mathrm{nM}$ DAPK siRNA 001; white bars, HHUA cells transfected with $50 \mathrm{nM}$ DAPK siRNA 002; ${ }^{*} \mathrm{p}<0.05,{ }^{* *} \mathrm{p}<0.01$. (B) Recombinant human soluble TRAIL suppresses the cell viability of HHUA cells in a dose-dependent manner; ${ }^{* * *} \mathrm{p}<0.001$.

the mechanism underlying the apoptosis induced by DAPK knockdown, we performed RNase protection assays of apoptosis-related genes in HHUA cells transfected with the DAPK siRNAs or control siRNA. As shown in Fig. 6, DAPK
siRNA transfections increased the mRNA levels of death receptor-mediated signaling molecules such as Fas, DcR1, DR5, DR4 and TRAIL in the DAPK siRNA-transfected HHUA cell. Of note, the expression levels of the death 
receptor DR4 and DR5 mRNAs and, in particular, TRAIL mRNA were simultaneously and remarkably elevated. In addition, the mRNA expression of an antagonistic decoy receptor, DcR1, was also slightly elevated. In the contrast, despite the increased mRNA expression of Fas, Fas ligand mRNA expression was not detected.

Suppression of endogenous DAPK expression in HHUA cells stimulates TRAIL protein expression. Next, we determined whether the level of TRAIL protein expression was elevated in the DAPK siRNA-transfected HHUA cells compared with the control siRNA-transfected cells, consistent with the findings for TRAIL mRNA obtained in the RNase protection assays. ELISAs based on cell lysates were utilized to assess the TRAIL protein levels. The results revealed that the DAPK siRNA-transfected HHUA cells expressed 5- to 10-fold higher levels of TRAIL protein than the control siRNA-transfected cells during the first $24-48 \mathrm{~h}$ after transfection, although these levels decreased thereafter (Fig. 7A). To further verify the possibility that the apoptosis observed in the DAPK siRNAtransfected cells was mediated through a TRAIL-mediated apoptotic signaling pathway, we investigated the susceptibility of HHUA cells to TRAIL. As expected, treatment of HHUA cells with recombinant soluble human TRAIL suppressed the cell viability in a dose-dependent manner (Fig. 7B), suggesting that HHUA cells express functional TRAIL receptors on their cell surface.

\section{Discussion}

This is the first report to show that DAPK may be a strong candidate molecule for a targeted anticancer therapy for human endometrial adenocarcinoma cells. DAPK is a $\mathrm{Ca}^{2+}$ calmodulin-dependent cytoskeleton-localized serine/threonine kinase that functions as a positive or negative mediator of apoptosis triggered by various apoptotic stimuli (16-23,26,27). To elucidate the role of endogenous DAPK in human endometrial adenocarcinoma cells, we examined the effects of suppression of endogenous DAPK expression after transfection of specific DAPK siRNAs into HHUA cells, which are welldifferentiated endometrial carcinoma cells. HHUA cells were reported to express higher levels of DAPK protein than several ovarian and uterine carcinoma cell lines and the normal human endometrium (28). The present findings revealed that the suppression of endogenous DAPK expression by the specific DAPK siRNAs induced TRAIL-mediated caspasedependent apoptosis in HHUA cells, indicating an essential role for endogenous DAPK in HHUA cell survival. Taken together, these results suggest that DAPK may represent a potential target for molecularly targeted cancer therapies.

The DAPK siRNA transfections induced remarkable expression of TRAIL mRNA and, to lesser extents, DR4 and DR5 mRNA expressions. The increased expression levels of DR4 and DR5 may have enhanced the apoptotic signals in response to the increased TRAIL expression induced by the DAPK siRNA transfections. We further found that the DAPK siRNA-transfected HHUA cells secreted large amounts of TRAIL protein and that treatment of HHUA cells with recombinant soluble human TRAIL suppressed the cell viability in a dose-dependent manner. Taken together, these data indicate that the suppression of endogenous DAPK expression in the
DAPK siRNA-transfected HHUA cells mainly induced apoptosis through a TRAIL-mediated apoptotic signaling pathway in an autocrine fashion. There is a recent report that synergistic cotreatment of tumor cells with TRAIL and chemotherapeutic drugs exerts cytotoxic effects on tumor cells via increased DR5 expression (30). Therefore, DAPK siRNA transfection could be useful as a combination therapy with anticancer chemotherapy for patients with multidrug-resistant cancers. Accordingly, DAPK may represent a novel candidate molecule for molecularly targeted anticancer therapies for human TRAIL receptor-expressing cancer cells, presumably through activation of the TRAIL-TRAIL receptor signaling pathway.

The targets for molecularly targeted anticancer therapies must have high specificity for the cancer cells. DAPK is highly expressed in differentiated human endometrial adenocarcinoma cells (28). Moreover, the systemic adverse effects of DAPK siRNA treatment on individuals are thought to be tolerable because DAPK-mutant mice develop into adults in a similar manner to normal mice and can reproduce (unpublished data). Therefore, DAPK may be a powerful candidate molecule for a targeted anticancer therapy for advanced endometrial adenocarcinomas.

\section{References}

1. Ashkenazi A and Dixit VM: Death receptors: signaling and modulation. Science 281: 1305-1308, 1998.

2. Pitti RM, Marsters SA, Ruppert S, Donahue CJ, Moore A and Ashkenazi A: Induction of apoptosis by Apo-2 ligand, a new member of the tumor necrosis factor cytokine family. J Biol Chem 271: 12687-12690, 1996.

3. Wiley SR, Schooley K, Smolak PJ, Din WS, Huang CP, Nicholl JK, Sutherland GR, Smith TD, Rauch C and Smith CA: Identification and characterization of a new member of the TNF family that induces apoptosis. Immunity 3: 673-682, 1995.

4. Sheridan JP, Marsters SA, Pitti RM, Gurney A, Skubatch M, Baidwin D, Ramakrishnan L, Gray CL, Baker K, Wood WI, Goddard AD, Godowski P and Ashkenazi A: Control of TRAILinduced apoptosis by a family of signaling and decoy receptors. Science 277: 818-821, 1997

5. Chaudhary PM, Eby M, Jasmin A, Bookwalter A, Murray J and Hood L: Death receptor 5, a new member of the TNFR family, and DR4 induce FADD-dependent apoptosis and activate the NF-kappaB pathway. Immunity 7: 821-830, 1997.

6. Pan G, Ni J, Wei YF, Yu G, Genze R and Dixit VM: An antagonist decoy receptor and a death domain-containing receptor for TRAIL. Science 277: 815-818, 1997.

7. Wu GS, Burns TF, McDonald ER, Jiang W, Meng R, Krantz ID, Kao G, Gan DD, Zhou JY, Muschel R, Hamilton SR, Spinner NB, Markowitz S, Wu G and El-Deiry WS: KILLER/DR5 is a DNA damage-inducible p53-regulated death receptor gene. Nat Genet 17: 141-143, 1997.

8. Ashkenazi A, Pai RC, Fong S, Leung S, Lawrence DA, Marsters SA, Blackie C, Chang L, McMurtrey AE, Hebert A, DeForge L, Koumenis IL, Lewis D, Harris L, Bussiere J, Koeppen H, Shahrokh Z and Schwall RH: Safety and antitumor activity of recombinant soluble Apo2 ligand. J Clin Invest 104: 155-162, 1999.

9. Walczak H, Miller RE, Ariail K, Gliniak B, Griffith TS, Kubin M, Chin W, Jones J, Woodward A, Le T, Smith C, Smolak P, Goodwin RG, Rauch CT, Schuh JC and Lynch DH: Tumoricidal activity of tumor necrosis factor-related apoptosis-inducing ligand in vivo. Nat Med 5: 157-163, 1999.

10. Leverkus M, Neumann M, Mengling T, Rauch CT, Brocker EB, Krammer PH and Walczak H: Regulation of tumor necrosis factor-related apoptosis-inducing ligand sensitivity in primary and transformed human keratinocytes. Cancer Res 60: 553-559, 2000.

11. Ichikawa K, Liu W, Zhao L, Wang Z, Liu D, Ohtsuka T, Zhang H, Mountz JD, Koopman WJ, Kimberly RP and Zhou T: Tumoricidal activity of a novel anti-human DR5 monoclonal antibody without hepatocyte cytotoxicity. Nat Med 7: 954-960, 2001. 
12. Hao C, Beguinot F, Condorelli G, Trencia A, van Meir EG, Yong VW, Parney IF, Roa WH and Petruk KC: Induction and intracellular regulation of tumor necrosis factor-related apoptosisinducing ligand (TRAIL) mediated apoptosis in human malignant glioma cells. Cancer Res 61: 1162-1170, 2001.

13. Nicholson DW and Thornberry NA: Life and death decisions. Science 299: 214-215, 2003.

14. Gross A, Yin XM, Wang K, Wei MC, Jockel J, Milliman C, Erdjument-Bromage H, Tempst P and Korsmeyer SJ: Caspase cleaved BID targets mitochondria and is required for cytochrome c release, while BCL-XL prevents this release but not tumor necrosis factor-R1/Fas death. J Biol Chem 274: 1156-1163, 1999.

15. Li H, Zhu H, Xu CJ and Yuan J: Cleavage of BID by caspase 8 mediates the mitochondrial damage in the Fas pathway of apoptosis. Cell 94: 491-501, 1998.

16. Deiss LP, Feinstein E, Berissi H, Cohen O and Kimchi A: Identification of a novel serine/threonine kinase and a novel $15-\mathrm{kD}$ protein as potential mediators of the gamma interferoninduced cell death. Genes Dev 9: 15-30, 1995.

17. Cohen O, Feinstein E and Kimchi A: DAP kinase is a $\mathrm{Ca}^{2+}$ calmodulin-dependent, cytoskeleton-associated protein kinase with cell death-inducing functions that depend on its catalytic activity. EMBO J 16: 998-1008, 1997.

18. Cohen O, Inbal B, Kissil JL, Raveh T, Berissi H, SpivakKroizaman T, Feinstein E and Kimchi A: DAP kinase participates in TNF-alpha- and Fas-induced apoptosis and its function requires the death domain. J Cell Biol 146: 141-148, 1999.

19. Jang CW, Chen CH, Chen CC, Chen JY, Su YH and Chen RH: TGFB induces apoptosis through Smad-mediated expression of DAP kinase. Nat Cell Biol 4: 51-58, 2002.

20. Raveh T, Droguett G, Horwitz MS, De Pinho RA and Kimchi A: DAP kinase activates a p19ARF/p53-mediated apoptotic checkpoint to suppress oncogenic transformation. Nat Cell Biol 3: 1-7, 2001.

21. Pelled D, Raveh T, Riebeling C, Fridkin M, Berissi $H$, Futerman AH and Kimchi A: Death-associated protein (DAP) kinase plays a central role in ceramide induced apoptosis in cultured hippocampal neurons. J Biol Chem 277: 1957-1961, 2002.
22. Yamamoto M, Hioki T, Nakajima-Iijima S and Uchino S: DAP kinase activity is critical for C (2)-ceramide-induced apoptosis in PC12 cells. Eur J Biochem 269: 139-147, 2002.

23. Wang WJ, Kuo JC, Yao CC and Chen RH: DAP kinase induces apoptosis by suppressing integrin activity and disrupting matrix survival signals. J Cell Biol 159: 169-179, 2002.

24. Kissil JL, Feinstein E, Cohen O, Jones PA, Tsai YC, Knowles MA, Eydmann ME and Kimchi A: DAP kinase loss of expression in various carcinoma and B-cell lymphoma cell lines: possible implications for role as tumor suppressor gene. Oncogene 15: 403-407, 1997.

25. Inbal B, Cohen O, Polak-Charcon S, Kopolovic J, Vadai E, Eisenbach L and Kimchi A: DAP kinase links the control of apoptosis to metastasis. Nature 390: 180-184, 1997.

26. Jin Y, Blue EK, Dixon S, Hou L, Wysolmerski RB and Gallagher PJ: Identification of a new form of death-associated protein kinase that promotes cell survival. J Biol Chem 276: 39667-39678, 2001.

27. Jin Y and Gallagher PJ: Antisense depletion of death-associated protein kinase promotes apoptosis. J Biol Chem 278: 51587-51593, 2003.

28. Bai T, Tanaka T, Yukawa K and Umesaki N: Reduced expression of death-associated protein kinase in human uterine and ovarian carcinoma cells. Oncol Rep 11: 661-665, 2003.

29. Ishiwata I, ishiwata C, Soma M, Arai J and Ishikawa H: Establishment of human endometrial adenocarcinoma cell line containing estradiol-17 beta and progesteron receptors. Gynecol Oncol 17: 281-290, 1984.

30. Nalgane M, Pan G, Weddle JJ, Dixit VM, Cavenee WK and Huang HJ: Increased death receptor 5 expression by chemotherapeutic agents in human gliomas causes synergistic cytotoxicity with tumor necrosis factor-related apoptosis-inducing ligand in vitro and in vivo. Cancer Res 60: 847-853, 2000. 